\title{
Synergistic Effects of Salts and Carbon Dioxide on Dermatophilus dermatonomus
}

\author{
By D. S. ROBERTS \\ Division of Animal Health, C.S.I.R.O., \\ McMaster Laboratory, Glebe, N.S.W., Australia
}

(Received 10 June 1964)

\begin{abstract}
SUMMARY
The addition of $\mathrm{K}$ and Na salts to cultures of Dermatophilus dermatonomus potentiated the effect of carbon dioxide $\left(\mathrm{CO}_{2}\right)$ in stimulating hyphal growth and delaying sporulation; the salts had virtually no effect in the absence of $\mathrm{CO}_{2}$. The maximum production of these effects required the same minimum concentration, about 50 m-equiv. cation/1., of a comparably wide range of salts, as did maximum alteration of the chemotactic response of the zoospores to $\mathrm{CO}_{2}$. It is suggested that there may be a common mechanism by which salts sensitize both the zoospores and the growing stages to $\mathrm{CO}_{2}$. If these reactions are induced by the salts and $\mathrm{CO}_{2}$ in the skin of infected sheep, they would partly account for the observed rapidity of both hyphal penetration and the emergence of zoospores.
\end{abstract}

\section{INTRODUCTION}

Carbon dioxide has been shown to affect both the growth of the actinomycete Dermatophilus dermatonomus, and the movement of its zoospores. Roberts (1963a) described a negative aerotactic response in which the zoospores moved away from the air/liquid interface in media of low salt content, but accumulated at the interface in media containing 40 or more m-equiv. monovalent salts/l. Roberts (1963 c) showed that the movement away from the interface was migration towards an optimum concentration of endogenous $\mathrm{CO}_{2}$. It seems likely that the salts added sensitized the zoospores to $\mathrm{CO}_{2}$ so that they migrated towards a lower optimum concentration, which they found near the interface where $\mathrm{CO}_{2}$ was lost by diffusion into the atmosphere. In cultures of $D$. dermatonomus Roberts (1963 $d$ ) showed that $\mathrm{CO}_{2}$ stimulated germination and growth but delayed development so that sporulation was inhibited and longer hyphae were formed. The present paper describes experiments which indicate that the effects of $\mathrm{CO}_{2}$ on germination, growth and development are potentiated by concentrations of salts similar to those affecting the chemotactic response of the zoospores.

\section{METHODS}

Organism. The two strains of Dermatophilus dermatonomus used were strains 18 and 47 of Roberts $(1963 d)$.

Liquid medium. $0.5 \%$ (w/v) Oxoid Lab-Lemco beef extract, 1.0\% (w/v) Difco Bacto or Proteose peptone, $0.1 \%$ (w/v) Difco yeast extract, and $0.15 \%(w / v)$ glucose, 
were dissolved in distilled water; $\mathrm{NaOH}$ was added so that the medium was at pH $7 \cdot 2$ after autoclaving $\left(120^{\circ}, 20 \mathrm{~min}\right.$.).

The growth of cultures with agitation. Cultures were sown and incubated by the methods described by Roberts $(1963 d)$. When sporulation was studied $45 \mathrm{ml}$. lots of liquid medium in 1 l. Erlenmeyer flasks were inoculated and incubated at $37^{\circ}$ overnight without agitation to produce a light growth of mycelium. The salts to be tested, or water in the case of controls, were then added to the medium in $5 \mathrm{ml}$. volumes, together with $1 \mathrm{ml}$. volumes of $50 \%(\mathrm{w} / \mathrm{v})$ peptone to ensure a sufficiency of nutrients during sporulation.

Before the flasks were sealed, the appropriate concentration of $\mathrm{CO}_{2}$ was added to the air space. In the case of cultures to be grown without $\mathrm{CO}_{2}, 5 \mathrm{ml} .50 \% \mathrm{NaOH}$ was added to a test tube standing within the flask to trap $\mathrm{CO}_{2}$ formed by the culture; $5 \mathrm{ml}$. water was added to the tubes in the flasks to which $\mathrm{CO}_{2}$ was added, so that the tubes in all flasks would have the same weight and therefore, since they were free to move as the flasks rotated, the same tendency to disturb the swirling medium. The cultures were then incubated with agitation at $27^{\circ}$ overnight.

When early filamentous growth was to be measured, the flasks again contained $45 \mathrm{ml}$. medium $+5 \mathrm{ml}$. of the appropriate sterile salt solutions. They were inoculated with standard volumes of suspensions of zoospores separated from the rest of the culture by passage through sterile 'Ekwip DS' filter pads (Industrial Equipment Australasia Pty. Ltd.). No extra peptone was added. The salt concentration and gaseous environment were adjusted at the time of inoculation. At the end of the incubation period growth was stopped by formalin (HCHO, $40 \%, \mathrm{w} / \mathrm{v}$ ) added to a final concentration of $0.5 \%(\mathrm{v} / \mathrm{v})$.

Growth of cultures without agitation. One-ml. volumes of solutions of $\mathbf{K}$ and $\mathrm{Na}$ salts were added to $9 \mathrm{ml}$. volumes of liquid medium in $1 \mathrm{oz}$. bottles, to give final concentrations of added cations of $0,25,50$, or 100 m-equiv./l. Dense suspensions of filtered zoospores were added in $0.2 \mathrm{ml}$. volumes and the cultures incubated with their lids unsealed at $37^{\circ}$ for $3 \mathrm{hr}$. Growth was then stopped by adding formalin to a final concentration of $1 \%(v / v)$. Differential counts were made to determine the proportion of the zoospores population which had budded at each salt concentration.

Reagents. The salts used were stated by the manufacturers to be of A.R. grade. The $\mathrm{CO}_{2}$ was obtained from cylinders filled by the Commonwealth Industrial Gases Ltd., Alexandria, N.S.W.

The measurement of growth. The extinction of zoospore suspensions was measured at $670 \mathrm{~m} \mu$ with a $1 \mathrm{~cm}$. light path in a Unicam S.P. 1400 spectrophotometer, after separation by filtration as described by Roberts (1963d). Filamentous growth was measured with the same instrument but after treatment of the culture with a homogenizer to disperse clumped hyphae.

The determination of differences due to synergism between salts and $\mathrm{CO}_{2}$. 'Synergism' is defined as the difference between the effect of $\mathrm{CO}_{2}$ and the salt when supplied together, and the sum of the effects of each when provided independently. It was measured in terms of extinction (E) as follows:

Difference due to synergism

$=$ effect of $\mathrm{CO}_{2}$ and salt together-effect of $\mathrm{CO}_{2}$ - effect of salt alone.

$=\left(\mathbf{E ~} \mathrm{CO}_{2}+\right.$ salt $-\mathrm{E}$ controls $)-\left(\mathrm{E} \mathrm{CO}_{2}-\mathrm{E}\right.$ controls $)-(\mathrm{E}$ salt $-\mathbf{E}$ controls $)$

$=\mathrm{E} \mathrm{CO}_{2}+$ salt $+\mathrm{E}$ controls $-\mathrm{E} \mathrm{CO}_{2}-\mathrm{E}$ salt. 
Where the effect of $\mathrm{CO}_{2}$ and salts was stimulatory, as in the case of mycelial growth, the synergism gave a positive value. In the case of sporulation the effect was inhibitory and a negative value was obtained.

The significance of the synergistic effect was determined by the application of Student's $t$ test to sums and differences, as described by Mather (1949). In some experiments there were significant differences in variance between groups of flasks receiving different treatments. The application of the method of Cochran \& Cox (1950) for $t$ tests on samples of different variance was found in each case to give the same result as a simple halving of the total number of degrees of freedom. Thus, to simplify tabulation, a corrected number of degrees of freedom has been given in these instances.

\section{RESULTS}

The effect of salts on the germination and budding of zoospores

In these experiments the cultures were not agitated during growth. The results in Table 1 show that $\mathrm{K}$ and $\mathrm{Na}$ salts had no appreciable effect on budding at 25 m-equiv. cation/l., but usually caused a statistically significant increase in the number of budded zoospores at 50 and 100 m-equiv./l. Although $\mathrm{CO}_{2}$ was not supplied there would have been endogenous $\mathrm{CO}_{2}$ present since the cultures were not agitated (Roberts, $1963 c$ ).

Table 1. The effect of salt concentration on the budding of zoospores of Dermatophilus dermatonomus in the presence only of endogenous $\mathrm{CO}_{2}$

\begin{tabular}{|c|c|c|c|c|c|c|c|}
\hline \multirow{2}{*}{$\begin{array}{l}\text { Strain of } \\
\text { D. derma- } \\
\text { tonomus }\end{array}$} & \multicolumn{5}{|c|}{$\begin{array}{l}\text { Salt concentration in } \\
\text { m-equiv, cation } / \mathrm{l} \text {. }\end{array}$} & \multirow[b]{2}{*}{$\begin{array}{l}\chi^{2} \text { for comparison of } \\
\text { pooled results at } 0\end{array}$} & \multirow{2}{*}{ Significance } \\
\hline & & \multicolumn{4}{|c|}{$\begin{array}{l}\text { No. of zoospores budded over } \\
\text { total counted }\end{array}$} & & \\
\hline 47 & $\mathrm{NaCl}$ & $\frac{56}{106}$ & $\frac{45}{100}$ & $\frac{67}{106}$ & $\frac{65}{110}$ & $5 \cdot 7$ & $P<0.02$ \\
\hline 18 & $\mathrm{NaCl}$ & $\frac{128}{257}$ & $\frac{125}{256}$ & $\frac{146}{253}$ & $\frac{146}{250}$ & $7 \cdot 46$ & $P<0.01$ \\
\hline 47 & $\begin{array}{c}\mathrm{Na}_{2} \mathrm{HPO}_{4}+ \\
\mathrm{NaH}_{2} \mathrm{PO}_{4} \\
(\mathrm{pH} \cdot \cdot 1)\end{array}$ & $\frac{56}{106}$ & $\frac{57}{101}$ & $\frac{69}{103}$ & $\frac{69}{106}$ & $5 \cdot 23$ & $P<0.05$ \\
\hline 47 & $\mathrm{Na}_{2} \mathrm{SO}_{4}$ & $\frac{56}{106}$ & $\frac{53}{100}$ & $\frac{46}{100}$ & $\frac{131}{206}$ & $7 \cdot 86^{*}$ & $P<0.01$ \\
\hline 47 & $\mathbf{K C l}$ & $\frac{113}{214}$ & $\frac{56}{102}$ & $\frac{69}{116}$ & $\frac{139}{219}$ & $4 \cdot 24$ & $P<0.95$ \\
\hline
\end{tabular}

* The results for $0, \mathbf{2 5}$, and $50 \mathrm{~m}$-equiv. cation/l. were pooled for comparison with those for 100 m-equiv./l.

The effect of salt concentration on hyphal growth in agitated cultures containing the same $\mathrm{CO}_{2}$ concentration

It was necessary to provide a relatively low $\mathrm{CO}_{2}$ concentration for effects of salt concentration to be demonstrable. Incubation was continued until there were measurable differences in extinction, but growth was stopped before sporulation 
began. These precautions were necessary because it was difficult to obtain a satisfactorily measurable difference in extinction, even when microscopy showed that the hyphae were of different length.

$\mathrm{NaCl}$ or $\mathrm{KBr}$ was added at concentrations extending from 0 to $\mathbf{7 0} \mathrm{m}$-equiv./1. Five flasks were used for each concentration. In the five experiments which fulfilled the necessary conditions, endogenous $\mathrm{CO}_{2}$ only was present in one, $0 \cdot 1 \%(v / v) \mathrm{CO}_{2}$ was added to the flasks in two, and $0.125 \%(\mathrm{v} / \mathrm{v}) \mathrm{CO}_{2}$ to the flasks in the other two. Incubation time was from 6 to $17 \mathrm{hr}$.

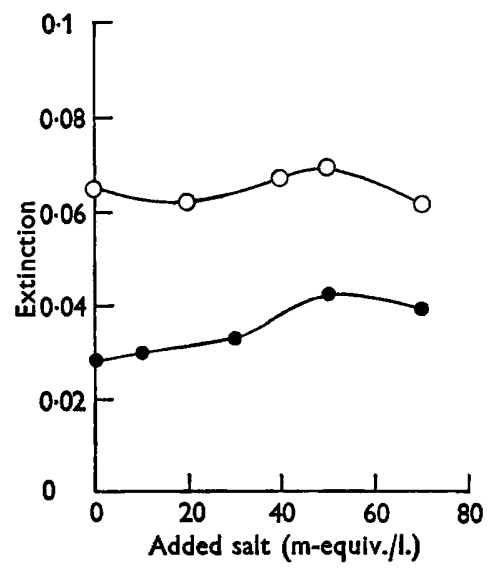

Fig. 1

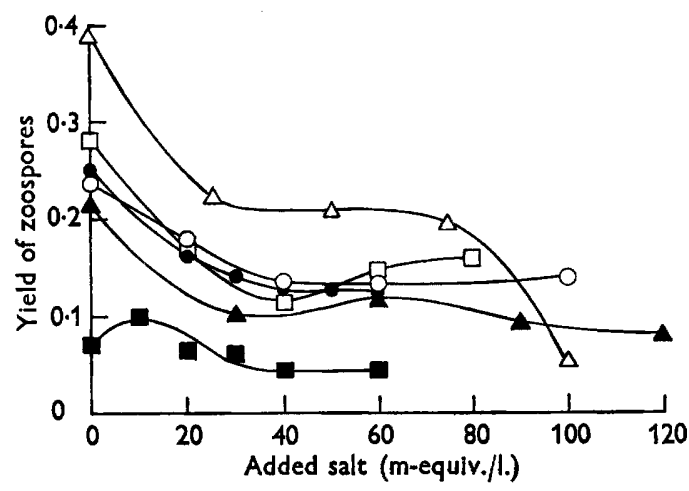

Fig. 2

Fig. 1. The effect of salt concentration on the early growth of D. dermatonomus in agitated cultures at $87^{\circ}$. The cultures were homogenized to disperse clumped hyphae before the extinction was measured. $O$, Sown with zoospores of strain 18 and supplied with $\mathrm{KBr}$ and $0.125 \%(\mathrm{v} / \mathrm{v}) \mathrm{CO}_{2}$ before incubation for $6 \mathrm{hr} ; 0$, strain 47 with $\mathrm{NaCl}$ and $0.1 \%(\mathrm{v} / \mathrm{v}) \mathrm{CO}_{2}$, and incubated for $11 \mathrm{hr}$.

Fig. 2. The effect of salt concentration on the sporulation of Dermatophilus dermatonomus strain 47 in agitated cultures. After an initial incubation without agitation at $87^{\circ}$ to produce mycelium, $1 \%(\mathrm{w} / \mathrm{v})$ peptone and the appropriate salt and $\mathrm{CO}_{2}$ concentrations were added to the flasks, which were then sealed and agitated overnight at $27^{\circ}$. Each culture was then divided into two portions. One was homogenized and used to measure the extinction (E) of the whole culture. The extinction of the other portion was measured after filtration to remove mycelium. The yield of zoospores (E) of filtrate/E of whole culture) was then calculated. $\triangle, \mathrm{NaCl}+0.5 \%(\mathrm{v} / \mathrm{v}) \mathrm{CO}_{2}, \Delta, \mathbf{K B r}+0.5 \%(\mathrm{v} / \mathrm{v})$ $\mathrm{CO}_{2}, \mathrm{O}$ and $\mathrm{O}, \mathrm{NaCl}+1 \%(\mathrm{v} / \mathrm{v}) \mathrm{CO}_{2}, \square, \mathrm{KBr}+2 \%(\mathrm{v} / \mathrm{v}) \mathrm{CO}_{2} ; \mathrm{G}, \mathrm{NaCl}+2 \%(\mathrm{v} / \mathrm{v}) \mathrm{CO}_{2}$.

The extinction was greatest in the flasks to which 40 m-equiv. salt/l. was added in two experiments, $50 \mathrm{~m}$-equiv./1. in two, and $60 \mathrm{~m}$-equiv./1. in the other. Figure 1 illustrates the growth response in the two experiments which had the briefest incubation, and in which the extinction thus depended most exclusively on hyphal length.

\section{The synergistic action of salts and $\mathrm{CO}_{2}$ on hyphal} growth in agitated cultures

To show synergistic effects of added salts and $\mathrm{CO}_{2}$ on hyphal growth it was necessary to fulfil the conditions stated in the preceding section. In each experiment four groups of 5 or 6 flasks were incubated together. One group (controls) had neither $\mathrm{CO}_{2}$ nor added salt in the medium; the second group had $\mathrm{CO}_{2}$ but no added 

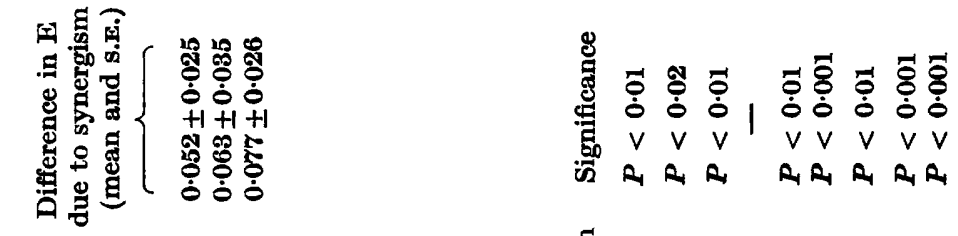

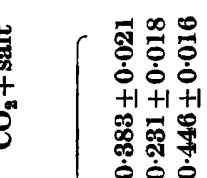

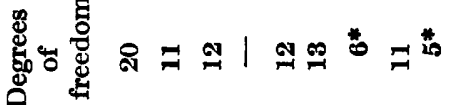

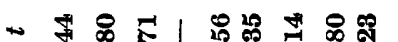
की

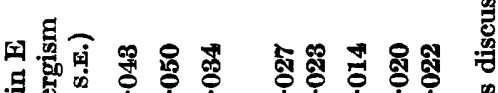

\&

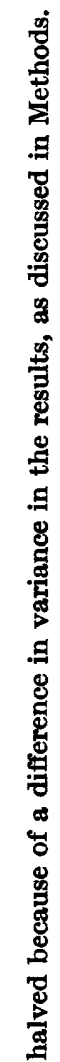

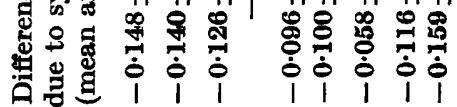

至兽

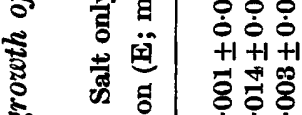

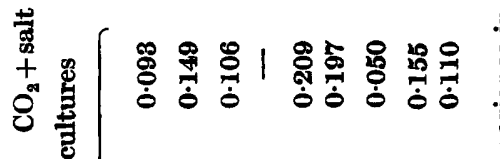

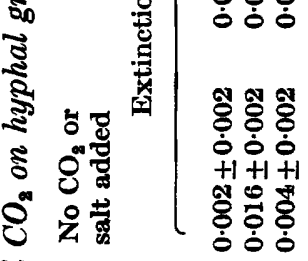

ॠ్

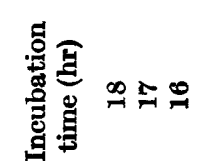

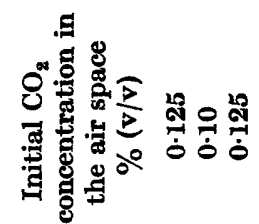

롱 离

ᄋी

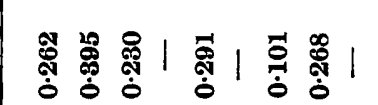

至国

농 或

$8^{2}$

胥

(1)

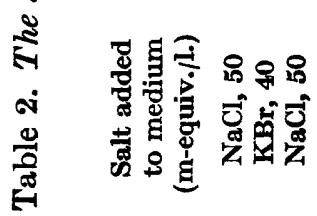

离

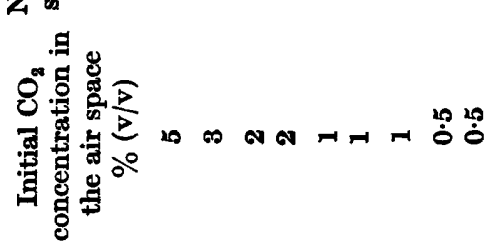

๓ 苛
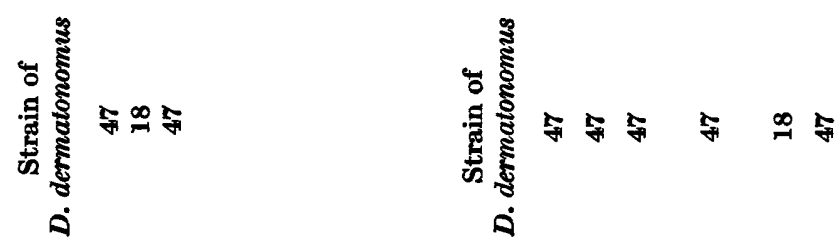
salt in the medium; the third had added salt but no $\mathrm{CO}_{2}$; the fourth had $\mathrm{CO}_{2}+$ salt, at the same concentrations as the flasks in the second and third groups, respectively.

In the absence of $\mathrm{CO}_{2}$, growth was slight and gave very low extinction readings. Microscopic examination showed that virtually all of the zoospores had budded but the resulting hyphae were very short. Added salts did not increase the extinction in the absence of $\mathrm{CO}_{2}$. The introduction of $\mathrm{CO}_{2}$ stimulated growth significantly. In the presence of $\mathrm{CO}_{2}$, added salts usually increased the extinction slightly. The synergism was appreciable in the three experiments illustrated in Table 2, but was statistically significant only in the third experiment shown $(t=2 \cdot 96$, with 9 degrees of freedom after adjustment as described in Methods; $p<0.02$ ).

\section{The effect of salt concentration on sporulation in agitated cultures containing the same $\mathrm{CO}_{2}$ concentration}

A significant effect of salt concentration on sporulation was much more easily measured than its effect on hyphal growth. It was possible to use higher $\mathrm{CO}_{2}$ concentrations because small differences in concentration had little effect in the absence of added salts. It was not necessary to adjust the incubation time. In the absence of $\mathrm{CO}_{2}$, sporulation was not affected by added salts. After the introduction of $\mathrm{CO}_{2}$, however, increases in salt concentration led to a marked decrease in the production of zoospores. Usually fewest zoospores were produced after the introduction of 40 m-equiv. cation/l.

In these experiments there was sometimes considerable variation in the total amount of growth, which was reflected in the number of zoospores produced. It was found, however, that the yield of zoospores (extinction of filtrate divided by extinction of homogenized whole culture) was not greatly affected by the variation in growth and conformed to a simple curve when plotted against salt concentration. The curves in Fig. 2 show that the minimum yield, reached after the addition of about 40 m-equiv. salt/l., was maintained when 1 or $2 \%(v / v) \mathrm{CO}_{2}$ had been supplied. With $0.5 \%(\mathrm{v} / \mathrm{v}) \mathrm{CO}_{2}$ there was a plateau rather than a minimum at this concentration.

\section{The synergistic action of salts and $\mathrm{CO}_{2}$ on sporulation in agitated cultures}

A statistically significant reduction in sporulation, due to synergism between added salts and $\mathrm{CO}_{2}$, was produced in each of the six experiments (Table 3).

\section{The permeability of growing forms to salts}

Since Roberts (1963a) showed that the chemotactic response of zoospores was altered only by salts to which they were permeable, the osmotic method used in the studies on zoospores was used to determine whether the growing stages were permeable to some of the salts which affected growth. The refractive index of the cytoplasm was measured by immersion refractometry, and relative changes in protoplasmic volume were calculated, as described by Roberts (1963a). At a total osmolar concentration of about $0 \cdot 1$, the average protoplasmic solids content of hyphae and dividing stages of strains 18 and 47 was $27 \%(w / v)$.

When the osmotic pressure was increased to 1 osmole by the addition of $\mathrm{NaCl}$ the average solids content of both strains was increased to $35 \%(w / v)$, indicating a 
$23 \%$ loss of volume. At 1.7 osmoles, with $\mathrm{Na}_{2} \mathrm{SO}_{4}$, the average solids content of both strains was greater than $44 \%(w / v)$, indicating a reduction in volume of more than $38 \%$. The increases in solids content were far too great to be accounted for by penetration of salt into the protoplasm. Moreover, the organisms were seen under the microscope to be smaller. There was no apparent restoration of volume or reduction in refractive index within $30 \mathrm{~min}$. with either salt, showing that the organisms had remained impermeable to them both.

\section{DISCUSSION}

In liquid media zoospores of Dermatophilus dermatonomus accumulate where the $\mathrm{CO}_{2}$ concentration is at an optimum (Roberts, $1963 \mathrm{c}$ ). Therefore the accumulation of zoospores at the air/liquid interface at increased salt concentrations (Roberts, $1963 a$ ) is probably due to a decrease in the optimum $\mathrm{CO}_{2}$ concentration so that the zoospores move to the interface, where the $\mathrm{CO}_{2}$ content is lowest as a result of diffusion into the atmosphere. The effect of the salt could be described as an increase in the sensitivity of zoospores to $\mathrm{CO}_{2}$ or, alternatively, as a potentiation of their response to $\mathrm{CO}_{2}$. Roberts $(1963 d)$ showed that $\mathrm{CO}_{2}$ stimulated hyphal growth but inhibited sporulation. In the experiments reported here, increased salt concentrations enhanced these effects. Thus salts seemed to sensitize the organism to $\mathrm{CO}_{2}$, or to potentiate the action of $\mathrm{CO}_{2}$ on the organism. This was supported by the fact that the salts had little or no effect in cultures from which all $\mathrm{CO}_{2}$ was rapidly eliminated, whereas in the presence of added $\mathrm{CO}_{2}$ increased salt concentration led to effects on growth significantly greater than the sum of the separate effects of $\mathrm{CO}_{2}$ and added salt.

There was also evidence that the effects of salt on chemotaxis and growth may be mediated by the same mechanism. Both actions were produced by a similar variety of salts. With chemotaxis the effect was produced by those mono- and divalent salts of the alkali metals to which the particular suspension of zoospores was permeable (Roberts, 1963a). In the present experiments, germination was stimulated by the chloride, sulphate, and mono- and dihydrogen orthophosphates of $\mathrm{Na}$, and by $\mathrm{KCl}$. Hyphal growth was stimulated, and sporulation inhibited, by $\mathrm{NaCl}$ and $\mathrm{KBr}$ (no other salts were tested). In addition, very similar concentrations were needed to produce the maximum effect on both chemotaxis and growth. Budding was not affected by 25 m-equiv. added salts/l. but, in four of five experiments, was stimulated to a maximum degree by $50 \mathrm{~m}$-equiv./l. In the case of hyphal growth, the maximum effect was at $40 \mathrm{~m}$-equiv./1. in two experiments, at 50 in two, and at 60 in the other one. A minimum yield of zoospores was always produced at about $40 \mathrm{~m}$-equiv./1. In all of these experiments the salt content of the medium before addition of the specific salts was about $8 \mathrm{~m}$-equiv./l., due mainly to $\mathrm{NaOH}$ added to adjust the $\mathrm{pH}$ value. It may thus be stated that at each stage the maximum effect on growth was produced by a minimum salt concentration of about 50 m-equiv. cation/l. Roberts $(\mathbf{1 9 6 3 a})$ observed that the chemotactic response was altered in only a few zoospores at 20 m-equiv. cation/l. but in virtually every zoospore in the suspension at $40 \mathrm{~m}$-equiv./l. A slight increase in effect on raising the salt concentration above $40 \mathrm{~m}$-equiv./l. was not observed, but would hardly have been recognizable under the experimental conditions. It may therefore be concluded that there was 
no real difference between the minimum concentration needed to give the greatest effect on the chemotactic response, and the minimum concentration for the greatest effect on growth.

The chemotactic response of zoospores was only altered by salts to which they were freely permeable (Roberts, $1963 a$ ), whereas the various growing stages were found to be impermeable to salts which had been shown to potentiate the effects of $\mathrm{CO}_{2}$ on growth. This casts doubt on the idea that there is a common synergistic mechanism in both cases. It is possible that such a mechanism operates in the interior of zoospores, but at the surface in growing stages. It seems more likely, however, that there are periods of permeability during growth which did not show under the conditions of the permeability experiment, and that the salts act within the organism in both phenomena.

According to Peters \& van Slyke (1931) the total concentration of $\mathrm{K}^{+}$and $\mathrm{Na}^{+}$ in animal tissue fluids is about $150 \mathrm{~m}$-equiv./1. This is well above the minimum that gives the maximum synergism with $\mathrm{CO}_{2}$ in vitro. If the salts and $\mathrm{CO}_{2}$ in the lesion which develops in infected sheep had the same action on Dermatophilus dermatonomus, the increased hyphal growth would contribute to the rapid penetration which has been observed after artificial infection. Also, sensitization to $\mathrm{CO}_{2}$ by salts in dried exudate on such lesions would accelerate the emigration of zoospores if, as discussed by Roberts $(1963 b, c)$, this is a response to the endogenous $\mathrm{CO}_{2}$ in the wetted scab.

Advice on the use of $t$ tests for differences due to interaction was kindly provided by Dr G. M. Tallis. I thank Mr H. H. Offord, Mrs J. Walker and Miss M. J. Crispe for their assistance with the experimental work.

\section{REFERENCES}

Cochran, W. G. \& Cox, G. M. (1950). Experimental Designs, p. 92. New York: Wiley.

Mather, K. (1949). Statistical Analysis in Biology. 3rd ed. p. 50. London: Methuen.

Peters, J. P. \& van SlyKe, D. D. (1931). Quantitative Clinical Chemistry. Volume I: Interpretations, p. 752: London: Baillière, Tindall and Cox.

Roberts, D. S. (1963a). Properties of Dermatophilus dermatonomus zoospores in relation to the transmission of mycotic dermatitis. Aust. J. agric. Res. 14, 373.

RoberTs, D. S. (1963b). The release and survival of Dermatophilus dermatonomus zoospores. Aust. J. agric. Res. 14, 386.

RoBerts, D.S. $(1963 c)$. Chemotactic behaviour of the infective zoospores of Dermatophilus dermatonomus. Aust. J. agric. Res. 14, 400.

Roberts, D. S. (1963d). The influence of carbon dioxide on the growth and sporulation of Dermatophilus dermatonomus. Aust. J. agric. Res. 14, 412. 